\title{
APLICACIÓN DEL MODELO CUANTITATIVO EN LA ELABORACIÓN DEL PROYECTO DE TESIS EN LAS FACULTADES DE DERECHO DE LIMA: 2010-2013
}

\section{APPLICATION OF THE QUANTITATIVE MODEL IN THE ELABORATION OF THE THESIS PROJECT IN THE FACULTIES OF LAW OF LIMA: 2010-2013}

\author{
Medardo Nizama Valladolid \\ Docente \\ Universidad Nacional Mayor de San Marcos \\ medardonizama@hotmail.com

\section{SUMARIO}

- Introducción

- Motivos o factores que determinan la aplicación del modelo cuantitativo

- Resultados de la investigación

- Conclusiones

\section{RESUMEN}

En este trabajo ofrecemos una visión conceptual y estadística de la aplicación del modelo cuantitativo en la preparación del proyecto de tesis en el postgrado. El objetivo propuesto del estudio fue determinar los motivos de aplicación del modelo cuantitativo en la elaboración del mismo proyecto de tesis.

Entre los resultados obtenidos se consideran que los motivos cognitivo-metodológico, motivacional-actitudinal y educativo-cultural, determinaron en las unidades participantes, la aplicación del modelo cuantitativo en la elaboración del referido proyecto de tesis en las facultades de Derecho: 2010-2013. Se aprecia que es el motivo cognitivo-metodológico el que determina, en medida significativa, en estudiantes y egresados, la aplicación de tal modelo, en razón a que los primeros han cursado asignaturas de Metodología de la Investigación y Seminario de Tesis; mientras que los segundos, no sólo han aprendido dichas materias, sino que también han recibido formación tutorial y en varios casos, experiencia estadístico-judicial. En lo que concierne a profesores, vemos que, en ambos Centros Superiores, son las competencias específicas las que propician la aplicación del modelo cuantitativo en la elaboración del proyecto de tesis.

En relación a las unidades participantes, fueron considerados 41 estudiantes, 166 egresados y 09 docentes sanmarquinos; y 112 estudiantes, 237 egresados y 12 profesores villarrealinos. La carencia de fuentes especializadas de consulta, la búsqueda de información de campo, y una intervención quirúrgica del suscrito, fueron algunas dificultades del trabajo.

\begin{abstract}
In this paper, we offer a conceptual and statistical view of the application of the quantitative model in the preparation of the postgraduate thesis project. The proposed objective of the study was to determine the reasons for applying the quantitative model in the preparation of the same thesis project.

Among the results obtained, the cognitivemethodological, motivational-attitudinal and educational-cultural reasons were determined in the participating units, the application of the quantitative model in the elaboration of the aforementioned thesis project in the Faculties of
\end{abstract}

1 Bachiller, Abogado, Magíster y Doctor por la UNMSM. Título de Profesor por la Universidad Nacional de Trujillo. Egresado de Maestría en Educación, Universidad Nacional Enrique Guzmán y Valle (La Cantuta), Docente Pre y Posgrado (UNMSM). 
Law: 2010-2013. It is appreciated that it is the cognitive-methodological reason that determines, to a significant extent, in students and graduates, the application of such a model, because the former have studied subjects of Research Methodology and Thesis Seminar; while the latter have not only learned these subjects, but have also received tutorial training and, in several cases, statistical-judicial experience. As far as teachers are concerned, we see that, in both Higher Centers, it is the specific competences that favor the application of the quantitative model in the elaboration of the thesis project.

In relation to the participating units, 41 students, 166 graduates and 09 San Marcos teachers were considered; and 112 students, 237 graduates and 12 Villarreal teachers. The lack of specialized sources of consultation, the search for field information, and a surgical intervention of the undersigned, were some difficulties of the work.

\section{PALABRAS CLAVE}

Modelo cuantitativo, elaboración de la de tesis, investigación jurídica, diseño cuantitativo, proyecto tesis jurídica.

\section{KEYWORDS}

Quantitative model, preparation of the thesis, legal research, quantitative design, legal thesis project.

\section{INTRODUCCIÓN}

Lo que se espera obtener de la aplicación de los resultados del presente estudio es contribuir en la solución del problema de escasez de tesis, un grave problema de las maestrías y doctorados del país, cuya gravedad causa preocupación entre las personas vinculadas a la docencia e investigación, a la administración de justicia y al ejercicio del derecho.

La realidad de esta carencia de tesis en los últimos años de acuerdo a las encuestas de opinión es crítica no obstante la idoneidad profesional de los docentes y su conocimiento e interés por la enseñanza del Derecho y la investigación. Entre las razones de tal carencia advertimos que ésta es compartida entre los protagonistas de dicha enseñanza y agentes de la comunidad académica y política, así como los medios de comunicación social masiva. Por un lado, el docente se muestra interesado por superar las deficiencias metodológicas y técnicas de la enseñanza, en consonancia con los cambios que entraña la aldea global; y por el otro lado, la motivación del educando por la mejora de su aprendizaje significativo $\mathrm{y}$ el interés en la preparación de sus memorias o monografías, así como incrementar la importante base de conocimientos del pregrado. Felizmente, está pasando el tiempo en que el educando mostraba poco interés intelectual por la lectura y la investigación. Ahora, el educando entiende la diferencia existente entre la forma como obtener el grado académico de magíster y cómo obtuvo el bachillerato y el título profesional. Actualmente, la preocupación por la enseñanza del Derecho es un tema presente y frecuente entre las personas vinculadas principalmente a la misma enseñanza jurídica, a la administración de justicia, al ejercicio de la defensa libre, etc. De igual modo, la comunidad académica y política piden buenas noticias sobre la calidad profesional y ética de estudiantes y profesores de postgrado de las facultades de Derecho del país

Obviamente, la carencia de tesis en el postgrado está ligada a la alta incidencia de escasez de recursos humanos altamente calificados en el mercado laboral, la misma que ha conllevado a los académicos a examinar las causas de dicha escasez, encontrando que una de ellas bien puede ser la falta de producción de proyectos de investigación en las escuelas y unidades de postgrado de Lima y del país, lo cual ha generado un impacto social que va en aumento ${ }^{2}$

Es por esta razón que nos alientan los resultados obtenidos en este trabajo, respecto de los mayores porcentajes alcanzados por el motivo o factor cognitivo-metodológico en estudiantes y egresados, así como también las competencias específicas que sobresalen en los docentes. También nos incentiva el hecho que en estos últimos años las autoridades de investigación, vienen recepcionando valiosas propuestas de estudio, que muestran una tendencia a la reactivación investigativa que debe monitorearse.

Con relación a las preguntas referimos que el problema central: ¿Qué factores determinan 2 Exposición realizada en el 19 de octubre 2015 en el
Auditórium Ella Dumbar Temple. Ciudad Universitaria UNMSM. 
la aplicación del modelo cuantitativo en la elaboración del proyecto de tesis en las facultades de Derecho de Lima: 2010-2013?, la respuesta de tal pregunta aparece en el enunciado de la hipótesis que aparece en el siguiente párrafo de esta misma introducción. Igualmente, damos cuenta que los objetivos se han cumplido a cabalidad al haberse analizado y explicado los tres motivos o factores que han determinado la aplicación del modelo cuantitativo en la elaboración del proyecto de tesis, tal como aparece en el desarrollo conceptual y estadístico de este trabajo. Respecto a la hipótesis referimos que se ha demostrado que los motivos o factores que han determinado la aplicación del modelo cuantitativo, son de carácter cognitivometodológico, motivacional-actitudinal y educativo-cultural, mientras que los profesores aplicaron las competencias específicas en su afán de propiciar la aplicación del modelo cuantitativo en la elaboración del proyecto de tesis; tal como aparece en los resultados consignados en el trabajo estadístico de este mismo trabajo, en el que aparecen las estimaciones porcentuales en cada una de las unidades de análisis debidamente muestreadas, respectivamente.

\section{MOTIVOS O FACTORES QUE DETERMINAN LA APLICACIÓN DEL MODELO CUANTITATIVO}

"El ser cultor de los saberes de la investigación jurídica formativa y avanzada; así como la difusión de sus resultados, es la misión esencial del estudiante del posgrado".

\section{Medardo Nizama V}

Está claro que hemos ingresado en una nueva sociedad denominada "era de la información", "sociedad del conocimiento" y de las "nuevas tecnologías", que no sólo transforman la ecología del aula, sino que también impulsan la aplicación de modelos o enfoques de trabajos en el contexto de una investigación formativa y/o avanzada.

La universidad ${ }^{3}$ entiende los procesos investigativos como situaciones que forman

3 Es decir, una comunidad académica orientada a la investigación y a la docencia, que brinda una formación humanística, científica y tecnológica con una clara conciencia de nuestro país como realidad multicultural. Ver art. $31 \mathrm{de}$ la Ley Universitaria No 30220, del 08 de junio del 2014. parte de la vida cotidiana del ser humano. Por ello, busca potenciar la capacidad de asombro del estudiante frente a la realidad, la búsqueda constante del conocimiento y la solución de problemas a lo largo de su formación humana y profesional.

La investigación formativa y/o avanzada se enmarca dentro de los cuatro pilares de la educación; esto es, aprender a conocer, aprender a ser, aprender a hacer y aprender a convivir, lo cual representa la base para el aprendizaje de formas creativas e innovadoras que fomenten en los estudiantes el pensamiento crítico, el interés por descubrir nuevos conocimientos, su capacidad y búsqueda constante del conocimiento ${ }^{4}$.

Precisamente, con el fin de fomentar el interés por la investigación en el estudiante de postgrado, hemos abordado tres estudios: el primero que analiza tres motivos o factores que determinan la aplicación del modelo cuantitativo (cognitivometodológico, motivacional-actitudinal y educativo-cultural); el segundo explica la elaboración del proyecto de tesis; y el tercero, analiza el planeamiento didáctico de la asignatura de Seminario de tesis.

\section{Primer motivo o factor cognitivo metodológico}

En este primer punto he intentado abordar temas de vasto alcance: ¿Cómo es que el hombre, cuyos contactos con el mundo son breves, personales y limitados logran, sin embargo, conocer tanto como conocen?

En verdad, aprender a construir conocimientos, constituye un nuevo desafío al ser humano y es en ese contexto en el que Gonzalo Ibarra 2006 y Lino Aranzamendi (2008) señalan lo siguiente: el primero indica que una primera forma de hacer metodología o epistemología, es considerarla como herramienta para pensar en una auténtica construcción del conocimiento jurídico, así como aprender los métodos y técnicas. Seguidamente, una segunda forma consiste en significarla como medio de reflexión para la transformación social, política y económica de los pueblos. La tercera forma se da cuando algunos jóvenes epistemólogos se preguntan sobre la posibilidad de construir conocimientos seguros desde la perspectiva del Derecho, en el que el objeto o razón de

4 Ver: Didáctica, tutoría e investigación formativa, material elaborado por el Vicerrectorado Académico de la UNMSM, pág. 49 
ser no sólo se considere la interpretación de las normas sino principalmente los contenidos sociales y políticos, en el contexto de un desarrollo humano sostenible.

Por lo demás, el profesor Lino Aranzamendi, en la misma línea del anterior autor, señala que al investigador de derecho le interesa no solo el estudio de la epistemología jurídica como sustento del conocimiento de la ciencia del derecho; sino también el estudio de los hechos o fenómenos de relevancia jurídicosocial. Así también expone que el objeto de la epistemología jurídica, es el estudio del significado, contenido, alcances, límites, métodos, orientaciones y expresiones de la cientificidad del derecho. Es por eso, que en esta disciplina se le considera como parte de la teoría de la ciencia jurídica donde se tratan los temas que se refieren a la cientificidad jurídica $\mathrm{y}$ sus formas.

La epistemología -concluye Aranzamendipermite al estudiante conocerla como procedimiento metodológico que permite no sólo comprender, descubrir, desarrollar, sino también interpretar o transformar el conocimiento sobre el derecho en la actualidad.

\section{Enfoques de la investigación}

Hernández Sampieri (2014) por su parte comenta que el enfoque cuantitativo utiliza la recolección de datos para probar hipótesis con base en la medición numérica y el análisis estadístico, con el fin de establecer pautas de comportamiento y probar teorías ${ }^{5}$. En el caso del enfoque cualitativo este utiliza la recolección y análisis de datos para afinar las preguntas de investigación o revelar nuevas interrogantes en el proceso de interpretación. Ambos enfoques son esenciales e imprescindibles; y constituyen una especie de guion, podríamos decir, para que el estudiante de Seminario de Tesis o cualquier educando de postgrado pueda utilizarlos en la elaboración de su proyecto.

\section{Definición de la investigación}

La ciencia está profundamente relacionada con la investigación. Si no hay investigación, simplemente la ciencia es nada. La realidad impone la obligación de no separar investigación y ciencia. Por eso, sin pretender

\footnotetext{
5 Ver el libro, Metodología de la investigación, del mismo Hernández Sampieri, página 4
}

superar las definiciones consignadas en los libros y revistas intentamos proponer una primera definición de Solís (2008), y él con la agudeza que le es peculiar aclara que la investigación es una "actividad humana por medio de la cual se producen nuevos conocimientos de nivel científico" . Una segunda definición de Hernández Sampieri indica que esta actividad representa un "conjunto de procesos sistemáticos, críticos y empíricos que se aplican al estudio de un fenómeno o problema". Ambas definiciones inspiran las páginas del presente trabajo.

\section{La investigación cuantitativa}

"Las teorías son redes, sólo quien lance cogerá"

Karl Popper ${ }^{7}$

En primer lugar, consideramos acertadas las ideas del profesor Aranzamendi (2015) cuando señala que a la ciencia solo le importa saber cómo se comprueban las hipótesis propuestas en la investigación, desprovistas de todo juicio valorativo. Es más, esta investigación pone especial interés en el estudio de la realidad como fruto de la causalidad; predice lo que resultará de la investigación; privilegia la recolección de datos medibles y repetibles, y en sus resultados se apoya en la estadística y en la selección de muestras significativas para generalizar sus conclusiones. La selección de la muestra es relevante para generalizar los resultados; de ahí que los casos particulares (individuales) si no presentan una población no tiene interés ${ }^{8}$.

En lo tocante a la dicotomía general arriba anotada Solís (2008) considera que las diferencias entre ambas investigaciones es que la primera referida a los estudios cuantitativos estos son generalizables, utilizan datos sólidos y repetibles, son estadísticamente representativos del universo y tienen una interpretación objetiva. Las características más apreciables de los estudios cualitativos son su comprensión del marco de referencia, su disponibilidad de datos ricos y profundos, y su interés en la descripción de los hechos. Estadísticamente, sin embargo, los estudios cualitativos no son representativos del universo

\footnotetext{
6 Solís Espinoza, ibidem, página 41.

7 Cita tomada del libro La lógica de la investigación científica de Karl Popper, pág. 13.

8 Consúltese la obra Investigación jurídica, de Lino Aranzamendi, página 152
} 
y por tanto no son generalizables. Por lo demás, están sujetos a una interpretación subjetiva y el método de observación que utilizan no es controlable"". Luego añade que ambos procedimientos se pueden complementar, según el objeto de investigación perseguido y de acuerdo al criterio del investigador. Por último expone que las investigaciones o diseños cuantitativos son los modelos más difundidos en las ciencias fácticas ${ }^{10}$.

\section{Segundo motivo o factor motivacional y actitudinal}

\section{Vocación pedagógica}

La idea que podemos formarnos sobre el papel del educador está en conexión íntima con la del papel de la educación. Sin embargo, conseguir y elegir una carrera o profesión, con un objetivo preciso y métodos modernos, no basta para asegurar el logro de un perfil profesional integral, con valores éticos, religiosos, de justicia, de personalidad, etc. (Peñaloza, 2000, viii). Igual acontece con los jóvenes de postgrado en razón de tener que elegir nuevamente la mención de su especialidad. La ausencia de la experiencia axiológica en el perfil del egresado, está dando al mundo de hoy sus frutos de indiferencia, insolidaridad, corrupción y violencia (Peñaloza, 2000, viii). Es menester - en ambos - un conjunto de aspiraciones y de aptitudes armoniosamente coordinadas, que constituye la vocación.

Hay que confesar que la situación de los jóvenes que se orientan hacia la investigación jurídica formativa $\mathrm{y} / \mathrm{o}$ avanzada, choca en la práctica con dificultades a la vez psicológicas y sociales: psicológicas, porque el carácter profesional del estudiante está muy mal definido, y aun cuando lo estuviera bien, no es el curso de la infancia ni siquiera en la época de la pubertad cuando podemos descubrir si el ser posee el conjunto de cualidades que lo constituyen; sociales, porque el estudiante puede dejarse seducir por consideraciones, tales como la ventaja pecuniaria, prestigio, etc.,

9 Solís Espinoza ibídem, página 94.

10 Este mismo autor, considerar cuatro investigaciones diseños cuantitativos, cada uno de los cuales presenta a su vez diversas variantes: Estudios experimentales; estudios causal comparativos (ex post facto), estudios correlacionales y estudios descriptivos. Asimismo, en las investigaciones en determinadas ciencias sociales como la antropologia y en algunas otras. Se pueden diferenciar tres modalidades: etnografia, estudios de casos e investigación acción. (Solís, pág. 94) que son enteramente extrañas a la naturaleza de su orientación profesional en postgrado.

\section{Motivación pedagógica}

Bien sabemos que la motivación pedagógica es el aprovechamiento del interés convertido en motivo del aprendizaje. En la escuela tradicional no se supo aprovechar el interés en la enseñanza porque se buscaba de modo indirecto y carecía de vitalismo. Felizmente, hoy esto ha cambiado con la escuela activa, la misma que concede un valor primordial al interés, como base de la motivación. En cada paso y en todo momento se tiene que buscar el punto de partida, o alguna fuerza impulsora del interés, que despierte vivamente la curiosidad, atención y energía del educando (Findlay). De donde surge el problema: ¿en dónde buscar los motivos o factores? Sin duda, éstos se encuentran en los intereses, objetivos y necesidades del alumno ${ }^{11}$ y que se revelan en el desarrollo de la clase en que el profesor descubrirá poco a poco en la misma clase o fuera de la misma. Conviene recordar que una vez que se tiene conocida la temática a enseñar es indispensable saber en qué dirección se va a encauzar el aprendizaje de los alumnos para la debida formación de los conocimientos y el desenvolvimiento de sus disposiciones ${ }^{12}$.

\section{Potencialidad humana}

Noblecilla Morán (2006), comentando este concepto escribe:

son las disponibilidades biogenéticas y neuronales que posee el ser humano para emprender labores mentales $y$ prácticas de toda naturaleza, orden, magnitud, profundidad, amplitud, etc. La potencialidad natural humana depende de la conformación y constitución orgánica, anatómica y fisiológica, que permite el mayor despliegue de actos mentales y motrices para el trabajo intelectual $y$ práctico. La potencialidad se expresa por la fuerza y energía vital. (Página 32)

\section{Capacidad humana}

El mismo autor comentando este segundo concepto básico a la letra expone:

\footnotetext{
11 Gonzales, Diego. Didáctica o dirección del aprendizaje; página 234.

$12 \mathrm{Al}$ igual que todo empeño humano, la tarea de educar tiene obvios límites y nunca cumple sino parte de sus mejores - ¡o peores!- propósitos. Véase "El valor de educar", de Fernando Savater, pág. 11.
} 
Son las progresivas posibilidades de ejecución laboral, cada vez más amplias, cada vez más profundas, cada vez de mayor complejidad y de exigencias físico-mentales. Cuando una persona tiene la seguridad de estar en capacidad de realizar algo progresivamente de mayor dificultad, es porque confía en sus potencialidades mentales y físicas que requiere la o las acciones. En la capacidad participa la voluntad de futuro, ésta es la intencionalidad de la capacidad; pero la capacidad también tiene límites que guardan relación con la constitución orgánica. (Página 33)

\section{Competencia}

Preciso es responder que las capacidades y competencias genéricas y específicas de los docentes universitarios, se fortalecen con el estudio, trabajo y capacitación permanente, puesto que tienen como función principal la investigación ${ }^{13}$. Desde esta perspectiva, llamo competencia al buen desempeño humano y profesional del profesor con el propósito de brindar una contribución real y efectiva para elevar el nivel de vida de la comunidad; y desde la perspectiva del educando, llamo competencia al buen accionar del educando, en sus labores académicas con el objeto de perfeccionar la formación profesional recibida en el pregrado. Así, también llamo competencia laboral, al buen desempeño del mismo educando, en sus labores que presta en su centro de trabajo, desarrollando una vida laboral intensa en la que resuelve problemas de la función a su cargo.

En definitiva, “posee competencia profesional quien dispone de los conocimientos, destrezas $\mathrm{y}$ actitudes necesarias para ejercer su propia actividad laboral, resuelve los problemas de forma autónoma y creativa y está capacitado para colaborar en su entorno laboral y en la organización del trabajo" (Echeverría, 2001, página 44).

\section{Motivos o caracteristicas del docente}

Con relación al uso de la tecnología de la información y la comunicación afirma Fernández Muñoz ${ }^{14}$ - el papel del profesor puede entenderse con la lectura del siguiente cuadro el mismo que especifica las características y tareas del profesor desde la perspectiva de dos modelos contrapuestos:

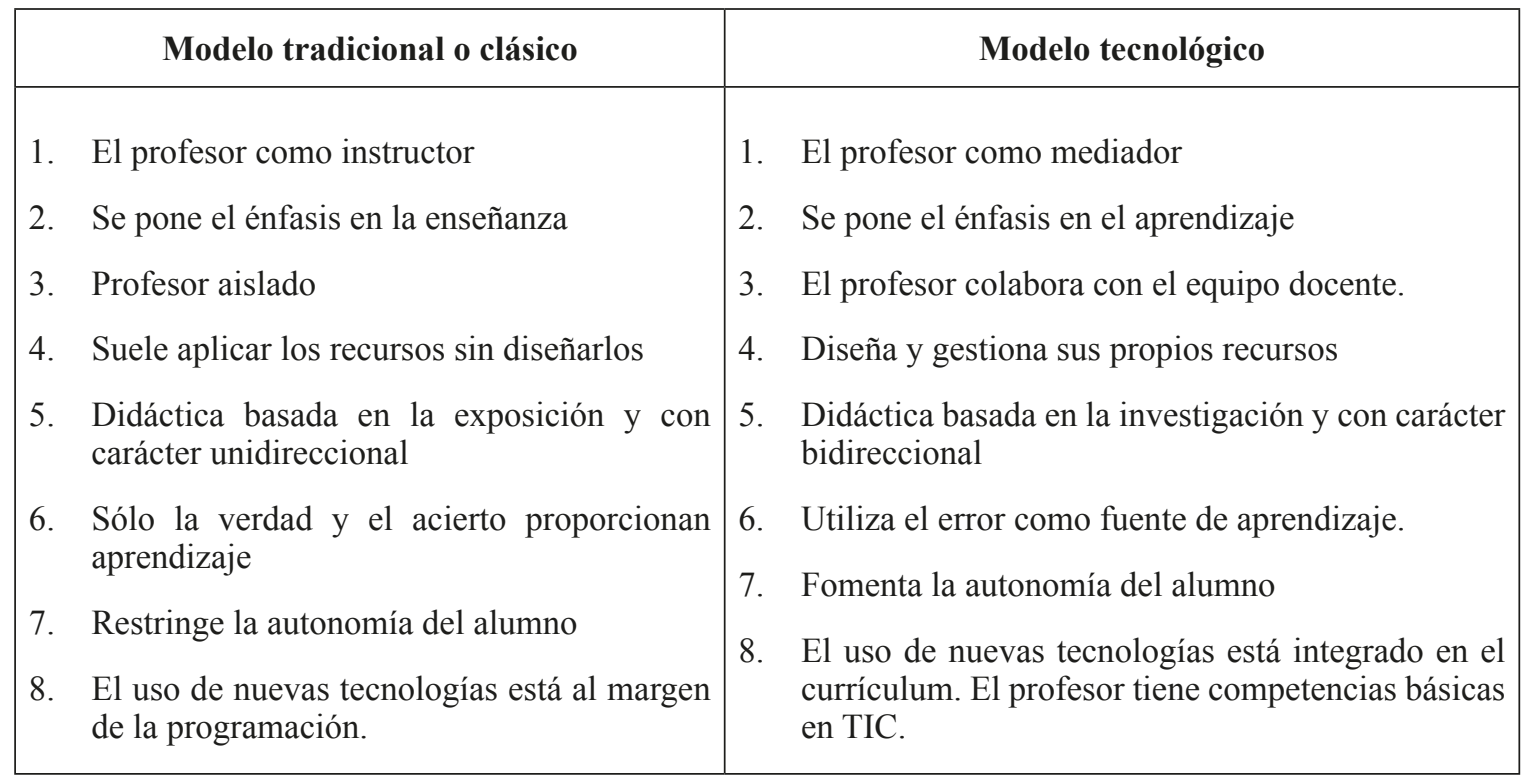

$13 \mathrm{El}$ artículo 79 de la Ley Universitaria $\mathrm{N}^{\circ} 30220$ establece que las funciones docentes son: la investigación, el mejoramiento continuo y permanente de la enseñanza, la proyección social y la gestión universitaria en los ámbitos que les corresponde.
14 Ver su trabajo denominado Competencias profesionales del docente en la sociedad del siglo XXI: 
El perfil del docente debiera configurarse como un profesional atento a todas las posibilidades de comunicación que el medio le ofrece, para hacer más adecuado, exitoso y atractivo el proceso de aprendizaje de los alumnos; un profesional que revise críticamente su propia práctica desde la reflexión de sus intervenciones como docente, y que pueda ayudar a sus alumnos para aprender a aprender en una sociedad cambiante y en constante evolución.

\section{Habilidades y destrezas del estudiante}

"Hay que ser zorro para conocer las trampas y león para hacer escapar a los lobos".

"El Príncipe" Nicolás de Maquiavelo Capítulo XVIII.

Ya hemos hablado de la importancia relativa a los motivos o características de los docentes, que es básica y necesaria, pero debemos hacer hincapié que lo que se pretende con el modelo cuantitativo es fundamentalmente el desarrollo de las habilidades y destrezas del estudiante. En efecto, los motivos o características aplicados a dicho modelo constituyen para nosotros la clave para estimular las habilidades o aptitudes del educando. Una clave de estímulo a tono con la práctica investigativa, inmersa en el proceso enseñanza-aprendizaje.

Howard Warren considera que la habilidad o aptitud es la condición o serie de características consideradas como síntomas de la capacidad de un individuo para adquirir, con un entrenamiento adecuado, algún conocimiento, habilidad o serie de reacciones, como la capacidad para aprender un idioma ${ }^{15} \mathrm{u}$ otros para comunicarnos mejor.

Entretanto NoblecillaMorán(2006) comentando el tema expone que las habilidades y destrezas corresponden a los alumnos y están dadas por sus propias potencialidades, capacidades, disposiciones, actitudes y experiencias. Gracias a dichas habilidades y destrezas el educando está en la posibilidad de compartir para efectuar cálculos y precisar resultados, ejercer dominio de técnicas de campo, etc.

Por su parte el profesor Mariano Moragues (1996), enfatiza que ninguna habilidad funciona aisladamente, sino que cada habilidad

15 Ver el Diccionario de Psicología, Howard C. Warren (Compilador), pág 20. cognitiva, cuando actúa pone en marcha a otras y se sirve de otras, de tal forma, que en cualquier acto intelectual interactúa un complejo sinnúmero de habilidades ${ }^{16}$.

\section{Tercer motivo o factor educativo cultural}

Pasamos ahora a ocuparnos del tercer y último motivo o factor de aplicación al modelo cuantitativo. Al igual que todo empeño humano la educación es un proceso humano y humanizador que tiene límites, pero no es una rutina simple. Actualmente tiene como sentido esencial el logro de un nuevo hombre: animador, creador e impulsor de avances en la búsqueda del cambio educativo, por mejores posibilidades de realización humana. Al nacer el hombre "trae consigo una riqueza biológica y psíquica y que es conformado por el medio en que le ha tocado vivir..."17.

Es la idea de educación, en sentido real y concreto $^{18}$, que creo va a tono con el proyecto de vida profesional del estudiante. Por cierto, es la nueva educación que Freire llama liberadora ${ }^{19}$, y que resulta fundamental para captar el formidable potencial humano. Un potencial que es la gran reserva y esperanza que tiene el país para construir el cambio educativo. El fin es lograr un Perú ahíto de ciencia, cultura e investigación. Un Perú con justicia, dignidad y cultura para todos los peruanos.

Desde el punto de vista formativo vemos que la educación jurídica hoy más que enseñar leyes es aprender el objeto de estudio y método del derecho entendido en su verdadero concepto científico. Precisamente, el derecho considerado como producto de la vida social ${ }^{20}$ y formando parte de la vida humana se sujeta a un continuo cambio y transformación. Por eso es que el sentido esencial de la nueva educación en derecho se orienta hacia el estudio de los principios universales y las normas del derecho, así como también la jurisprudencia, para proyectarlos en los casos concretos que presente la realidad social.

16 Ver su libro, Hacia la escuela posible, pág. 216

17 Cfr. Emilio Barrantes. Introducción a la Pedagogía, página 35

18 Ver Álvaro Villavicencio Whittembury. Acerca del concepto de educación. Cuestiones fundamentales de la Pedagogía, página 29.

19 Véase La obra, Pedagogía del oprimido, de Paulo Freire, página 4.

20 Vida humana, sociedad y derecho, de Luis Recaséns Siches, citado por Héctor Fix- Zamudio en su obra Metodología, docencia e investigación jurídicas, página 58 
Es significativa la preocupación actual sobre el prestigio científico del jurista, el mismo que se ha mellado frente a los avances de otras ciencias sociales. Sin duda alguna, la profesión del jurista ha sido y es objeto en el ánimo popular de las más encontradas opiniones, al punto que un autor español ha escrito un trabajo denominado "Oficio noble o diabólico" en el que trata las ideas contradictorias y las antinomias de la profesión jurídica. Sin embargo, y no obstante ello, vemos con satisfacción, que los estudios jurídicos continúan avanzando, tal como se aprecia en la bibliografía y otros documentos de las últimas décadas ${ }^{21}$

Decía Salazar Bondy que la vinculación de la educación nacional con el desarrollo económico y cultural del país es un tema capital de toda reflexión sobre los problemas educacionales, socio-jurídicos y socio-políticos en general ${ }^{22}$.

Por su parte, Emilio Barrantes, comentando el tema sobre educación y cultura afirma que ambos se

vinculan íntimamente al porvenir del hombre, hasta el punto de que algunos la consideran como una panacea para los pueblos cuyo retraso es evidente.

Paz y democracia, educación y cultura, son metas a que se aspira llegar, cada vez con mayor ansiedad a medida que crecen el odio, la opresión y el egoísmo, los bajos apetitos y las formas groseras de una vida impulsiva ${ }^{23}$.

\section{RESULTADOS DE LA INVESTIGACIÓN ${ }^{24}$}

\section{UNMSM}

ESTUDIANTES

\section{Gráfico 1}

Aplicación de los factores cognitivometodológicos del modelo cuantitativo en la elaboración del proyecto de investigación

\footnotetext{
21 Ibídem, página 57

22 Ver la obra, En torno a la educación, de Augusto Salazar Bondy, página 63

23 Ver la obra, Introducción a la pedagogía, de Emilio Barrantes, página 37.

24 Por razón de espacio solo se presentan los gráficos 1,2 y 3 que muestran los resultados de estudiantes de la UNMSM; y por la misma razón solo se presenta con tablas los resultados de egresados y profesores de la se presenta con
misma UNMSM.

misma UNMSM.
Todos las 18 tablas y gráficos del estudio fueron presentados Todos las 18 tablas y gráficos del estudio fueron presentados del 2016
}

en estudiantes de la Unidad Post Grado de la Facultad de Derecho de la UNMSM 2010-2013.

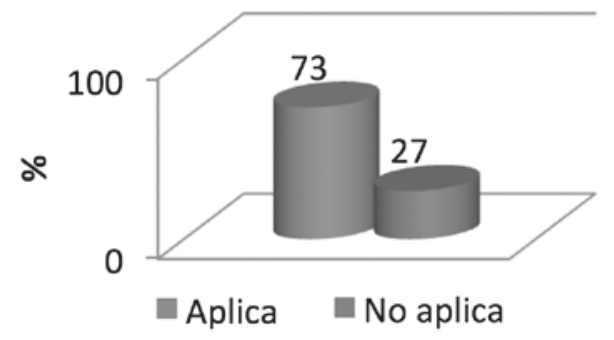

Del gráfico se aprecia que del total de estudiantes de la Unidad Post Grado de la Facultad de Derecho de la UNMSM, el 73\% aplica los factores cognitivo-metodológicos del modelo cuantitativo en la elaboración de su proyecto de investigación, mientras que el $27 \%$ no aplica los factores cognitivo-metodológico del modelo cuantitativo en la elaboración de su proyecto de investigación.

\section{Gráfico 2}

Aplicación de los factores motivacionalactitudinales del modelo cuantitativo en la elaboración del proyecto de investigación en estudiantes de la Unidad Post Grado de la Facultad de Derecho de la UNMSM.

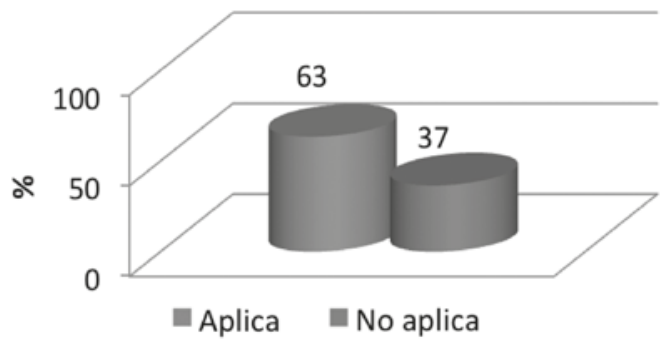

Del gráfico se aprecia que del total de estudiantes de la Unidad Post Grado de la Facultad de Derecho de la UNMSM, el 63\% aplica factores motivacional-actitudinales del modelo cuantitativo en la elaboración de su proyecto de investigación, mientras que el $37 \%$ no aplica los factores motivacionalactitudinales del modelo cuantitativo en la elaboración de su proyecto de investigación.

\section{Gráfico 3}

Aplicación de los factores educativo- culturales del modelo cuantitativo en la elaboración del proyecto de investigación en estudiantes de la 
Unidad Post Grado de la Facultad de Derecho de la UNMSM.

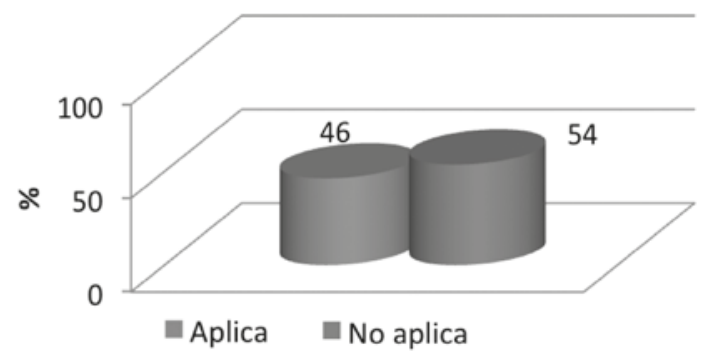

Del gráfico se aprecia que del total de estudiantes de la Unidad Post Grado de la Facultad de Derecho de la UNMSM, el 46\% aplica los factores educativo-culturales del modelo cuantitativo en la elaboración de su proyecto de investigación, mientras que el 54\% no aplica los factores educativo- culturales del modelo cuantitativo en la elaboración de su proyecto de investigación

\section{UNMSM}

EGRESADOS

\section{Tabla 1}

Aplicación de los factores cognitivometodológicos del modelo cuantitativo en la elaboración del proyecto de investigación en egresados de la Unidad Post Grado de la Facultad de Derecho de la UNMSM 20102013.

\begin{tabular}{|l|l|l|}
\hline & Cantidad & Porcentaje \\
\hline Aplica & 93 & $80 \%$ \\
\hline No aplica & 23 & $20 \%$ \\
\hline Cantidad & 116 & $100 \%$ \\
\hline
\end{tabular}

De la tabla se aprecia que del total de egresados de la Unidad Post Grado de la Facultad de Derecho de la UNMSM, el 80\% aplica los factores cognitivo-metodológicos del modelo cuantitativo en la elaboración de su proyecto de investigación, mientras que el $20 \%$ no aplica los factores cognitivo-- metodológicos del modelo cuantitativo en la elaboración de su proyecto de investigación.

\section{Tabla 2}

Aplicación de los factores motivacionalactitudinales del modelo cuantitativo en la elaboración del proyecto de investigación en egresados de la Unidad Post Grado de la Facultad de Derecho de la UNMSM.

\begin{tabular}{|l|l|l|}
\hline & Cantidad & Porcentaje \\
\hline Aplica & 79 & $68 \%$ \\
\hline No aplica & 37 & $32 \%$ \\
\hline Cantidad & 116 & $100 \%$ \\
\hline
\end{tabular}

De la tabla se aprecia que del total de egresados de la Unidad Post Grado de la Facultad de Derecho de la UNMSM, el 68\% aplica los factores motivacional-actitudinales del modelo cuantitativo en la elaboración de su proyecto de investigación, mientras que el $32 \%$ no aplica los factores motivacional-actitudinales del modelo cuantitativo en la elaboración de su proyecto de investigación.

\section{Tabla 3}

Aplicación de los factores educativo-culturales del modelo cuantitativo en la elaboración del proyecto de investigación en egresados de la Unidad Postgrado de la Facultad de Derecho de la UNMSM.

\begin{tabular}{|l|l|l|}
\hline & Cantidad & Porcentaje \\
\hline Aplica & 57 & $49 \%$ \\
\hline No aplica & 59 & $51 \%$ \\
\hline Cantidad & 116 & $100 \%$ \\
\hline
\end{tabular}

De la tabla se aprecia que del total de egresados de la Unidad de Post Grado de la Facultad de Derecho de la UNMSM, el 49\% aplica los factores educativo- culturales del modelo cuantitativo en la elaboración de su proyecto de investigación, mientras que el 51\% no aplica los factores educativo- culturales del modelo cuantitativo en la elaboración de su proyecto de investigación.

\section{UNMSM}

\section{PROFESORES}

\section{Tabla 1}

Aplicación de las competencias específicas en la elaboración del proyecto de investigación en profesores de la Unidad de Post Grado de Facultad de Derecho de la UNMSM según estudiantes 


\begin{tabular}{|l|l|l|}
\hline & Cantidad & Porcentaje \\
\hline Aplica & 32 & $78 \%$ \\
\hline No aplica & 9 & $22 \%$ \\
\hline Cantidad & 41 & $100,0 \%$ \\
\hline
\end{tabular}

De la tabla se aprecia que del total de profesores de la Unidad Post Grado de la Facultad de Derecho de la UNMSM, el 78\% opinan que los profesores aplica las competencias específicas en la orientación del proyecto de investigación, mientras que el $22 \%$ opinan que los profesores no aplica las competencias específicas en la en la orientación del proyecto de investigación

\section{Tabla 2}

Aplicación de las competencias genéricas en la elaboración del proyecto de investigación en profesores de la Unidad Post Grado de la Facultad de Derecho de la UNMSM

\begin{tabular}{|l|l|l|}
\hline & Cantidad & Porcentaje \\
\hline Aplica & 26 & $63 \%$ \\
\hline No aplica & 15 & $37 \%$ \\
\hline Cantidad & 41 & $100,0 \%$ \\
\hline
\end{tabular}

De la tabla se aprecia que del total de profesores de la Unidad Post Grado de la Facultad de Derecho de la UNMSM el $63 \%$ opinan que los profesores aplican las competencias genéricas en la orientación del proyecto de investigación, mientras que el $37 \%$ opinan que los profesores no aplican las competencias genéricas en la orientación proyecto de investigación.

\section{Tabla 3}

Aplicación de las competencias metodológicas en la elaboración del proyecto de investigación en profesores de la Unidad Post Grado de la Facultad de Derecho de la UNMSM.

\begin{tabular}{|l|l|l|}
\hline & Cantidad & Porcentaje \\
\hline Aplica & 6 & $67 \%$ \\
\hline No aplica & 3 & $33 \%$ \\
\hline Cantidad & 9 & $100,0 \%$ \\
\hline
\end{tabular}

De la tabla se aprecia que del total de profesores de la Unidad Post Grado de la Facultad de Derecho de la UNMSM, el 67\%

aplica las competencias metodológicas en la en la orientación del proyecto de investigación, mientras que el 33\% del total de profesores no aplica las competencias metodológicas en la orientación del proyecto de investigación.

\section{UNFV 25}

\section{ESTUDIANTES}

\section{Gráfico 1}

Aplicación de los factores cognitivometodológicos del modelo cuantitativo en la elaboración del proyecto de investigación en estudiantes de la EUPG de la Facultad de Derecho y Ciencia Política de la UNFV.

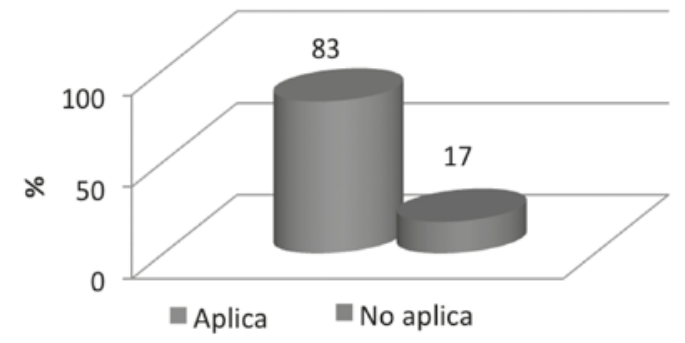

Del gráfico se aprecia que del total de estudiantes de la EUPG de la Facultad de Derecho y Ciencia Política de la UNFV, el 33\% aplica los factores cognitivo-metodológicos del modelo cuantitativo en la elaboración de su proyecto de investigación, mientras que el 17\% no aplica los factores cognitivo-metodológico del modelo cuantitativo en la elaboración de su proyecto de investigación.

\section{Gráfico 2}

Aplicación de los factores motivacionalactitudinal del modelo cuantitativo en la elaboración del proyecto de investigación en estudiantes de la EUPG. Facultad de Derecho y Ciencia Política de la UNFV.

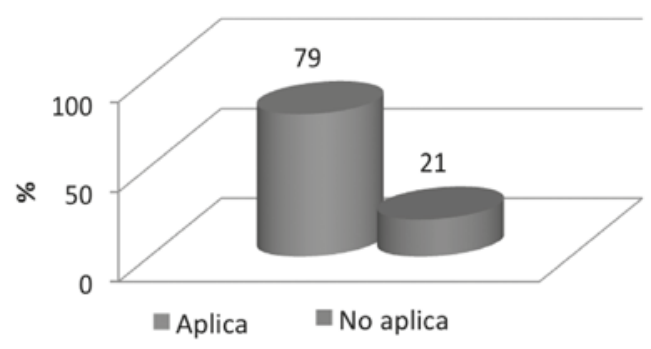

25 Por razón de espacio solo se presentan los gráficos 1,2 y 3 que muestran los resultados de estudiantes de la UNFV; $y$, por la misma razón solo se presenta con tablas los resultados de egresados y profesores de la misma UNFV. 
Del gráfico se aprecia que del total de estudiantes de la EUPG de la Facultad de Derecho y Ciencia Política de la UNFV, el 79\% aplica los factores motivacional-actitudinales del modelo cuantitativo en la elaboración de su proyecto de investigación, mientras que el $21 \%$ no aplica los factores motivacionalactitudinales del modelo cuantitativo en la elaboración de su proyecto de investigación.

\section{Gráfico 3}

Aplicación de los factores educativo- culturales del modelo cuantitativo en la elaboración del proyecto de investigación en estudiantes de la EUPG de la Facultad de Derecho y Ciencia Política de la UNFV.

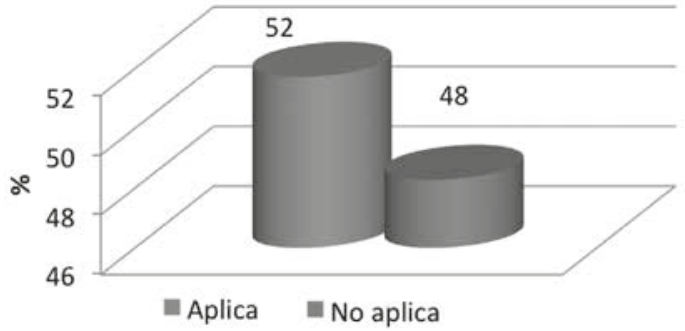

Del gráfico se aprecia que del total de estudiantes de la EUPG de la Facultad de Derecho y Ciencia Política de la UNFV, el $52 \%$ aplica los factores educativo-culturales del modelo cuantitativo en la elaboración de su proyecto de investigación, mientras que el $48 \%$ no aplica la factores educativo- culturales del modelo cuantitativo en la elaboración de su proyecto de investigación.

\section{UNFV}

EGRESADOS

\section{Tabla 1}

Aplicación de los factores cognitivometodológicos del modelo cuantitativo en la elaboración del proyecto de investigación en egresados de la EUPG de la Facultad de Derecho y Ciencia Política de la UNFV 2010-2013.

\begin{tabular}{|l|l|l|}
\hline & Cantidad & Porcentaje \\
\hline Aplica & 125 & $85 \%$ \\
\hline No aplica & 22 & $15 \%$ \\
\hline Cantidad & 147 & $169 \%$ \\
\hline
\end{tabular}

De la tabla se aprecia que del total de egresados de la EUPG de la Facultad de Derecho y Ciencia Política de la UNFV, el 85\% aplica los factores cognitivo- metodológicos del modelo cuantitativo en la elaboración de su proyecto de investigación, mientras que el $15 \%$ no aplica los factores cognitivo- metodológico del modelo cuantitativo en la elaboración de su proyecto de investigación.

\section{Tabla 2}

Aplicación de los factores motivacionalactitudinales del modelo cuantitativo en la elaboración del proyecto de investigación en egresados de la EUPG de la Facultad de Derecho y Ciencia Política de la UNFV.

\begin{tabular}{|l|l|l|}
\hline & Cantidad & Porcentaje \\
\hline Aplica & 110 & $75 \%$ \\
\hline No aplica & 37 & $25 \%$ \\
\hline Cantidad & 147 & $169 \%$ \\
\hline
\end{tabular}

De la tabla se aprecia que del total de egresados de la EUPG de la Facultad de Derecho y Ciencia Política de la UNFV, el 75\% aplica los factores motivacional-actitudinales del modelo cuantitativo en la elaboración de su proyecto de investigación, mientras que el $25 \%$ no aplica los factores motivacional- actitudinales del modelo cuantitativo en la elaboración de su proyecto de investigación.

\section{Tabla 3}

Aplicación de los factores educativo- culturales del modelo cuantitativo en la elaboración del proyecto de investigación en egresados de la EUPG de la Facultad de Derecho y Ciencia Política de la UNFV.

\begin{tabular}{|l|l|l|}
\hline & Cantidad & Porcentaje \\
\hline Aplica & 77 & $52 \%$ \\
\hline No aplica & 70 & $48 \%$ \\
\hline Cantidad & 147 & $169 \%$ \\
\hline
\end{tabular}

De la tabla se aprecia que del total de egresados de la EUPG de la Facultad de Derecho y Ciencia Política de la UNFV, el 52\% aplica los factores educativo- culturales del modelo 
cuantitativo en la elaboración de su proyecto de investigación, mientras que el $48 \%$ no aplica la factores educativo- culturales del modelo cuantitativo en la elaboración de su proyecto de investigación.

\section{UNFV}

\section{PROFESORES}

\section{Tabla 1}

Aplicación de las competencias específicas en la elaboración del proyecto de investigación en profesores de la EUPG de la Facultad de Derecho y Ciencia Política de la UNFV según estudiantes

\begin{tabular}{|l|l|l|}
\hline & Cantidad & Porcentaje \\
\hline Aplica & 73 & $84 \%$ \\
\hline No aplica & 14 & $16 \%$ \\
\hline Cantidad & 87 & $100 \%$ \\
\hline
\end{tabular}

De la tabla se aprecia que del total de profesores de la EUPG de la Facultad de Derecho y Ciencia Política de la UNFV, el $84 \%$ opinan que el profesor aplica las competencias específicas en la en la orientación del proyecto de investigación, mientras que el $16 \%$ opinan que el profesor no aplica las competencias específicas en la en la orientación del proyecto de investigación.

\section{Tabla 2}

Aplicación de las competencias genéricas en la elaboración del proyecto de investigación en profesores de la EUPG de la Facultad de Derecho y Ciencia Política de la UNFV.

\begin{tabular}{|l|l|l|}
\hline & Cantidad & Porcentaje \\
\hline Aplica & 59 & $68 \%$ \\
\hline No aplica & 28 & $32 \%$ \\
\hline Cantidad & 87 & $100 \%$ \\
\hline
\end{tabular}

De la tabla se aprecia que del total profesores de la EUPG de la Facultad de Derecho y Ciencia Política de la UNFV, el $68 \%$ opinan que los profesores aplican las competencias genéricas en la en la orientación del proyecto de investigación, mientras que el $32 \%$ opinan que los profesores no aplican las competencias genéricas en la orientación proyecto de investigación.

\section{Tabla 3}

Aplicación de las competencias metodológicas en la elaboración del proyecto de investigación en profesores de la EUPG de la Facultad de Derecho y Ciencia Política de la UNFV.

\begin{tabular}{|l|l|l|}
\hline & Cantidad & Porcentaje \\
\hline Aplica & 9 & $75 \%$ \\
\hline No aplica & 3 & $25 \%$ \\
\hline Cantidad & 12 & $14 \%$ \\
\hline
\end{tabular}

De la tabla se aprecia que del total de profesores de la EUPG de la Facultad de Derecho y Ciencia Política de la UNFV, el $75 \%$ aplica las competencias metodológicas en la en la orientación del proyecto de investigación, mientras que el $25 \%$ del total de profesores no aplica las competencias metodológicas en la orientación proyecto de investigación.

\section{Interpretación de datos}

Del análisis efectuado en base al marco teórico, antecedentes del estudio, problema y trabajo de medición, vemos que la hipótesis principal ha sido comprobada, gracias a la acción de las unidades participantes. También fue posible encontrar que en estudiantes y egresados es el factor cognitivo-metodológico, el que tiene mayores porcentajes de aplicación y respecto a los docentes, se encontró que son las competencias específicas las que mayormente propician la aplicación del modelo cuantitativo, en la elaboración del proyecto de tesis; en ambos centros de educación superior. Dicho hallazgo coincide con la afirmación de Gonzalo Ibarra, quien expone que aprender a construir conocimientos (lo cognitivo) constituye un nuevo desafío al ser humano y que lo epistemológico significa un medio de reflexión para la transformación social. Igualmente, Lino Aranzamendi señala que el epistemológico permite al estudiante conocerlo como procedimiento metodológico que permite comprender, descubrir, desarrollar, interpretar o transformar el conocimiento ${ }^{26}$.

Por su parte Popper considera que,

la ciencia no es un sistema de enunciados seguros y bien asentados, ni uno que avance firmemente hacia un estado final. Nuestra

26 ARANZAMENDI, LINO. Epistemología y la investigación cualitativa cuantitativa en el Derecho, 45. 
ciencia no es conocimiento (episteme); nunca puede pretender que ha alcanzado la verdad, ni siquiera el sustituto de ésta que es la probabilidad ${ }^{27}$.

Desde luego, los otros dos motivos o factores, tales como el motivacional-actitudinal y educativo-cultural, en mi opinión, su relevancia estriba en que los docentes para impartir las disciplinas de su especialidad, siempre, es necesario una formación motivacional $y$ actitudinal, así como también una formación educativo-cultural, en razón de que un aspecto importante en la aplicación de los métodos, estrategias y técnicas didácticas, es el desarrollo de una relación entre profesor-alumno apropiada; tal como aparece consignado en el punto de la motivación pedagógica de este trabajo.

En cuanto a la formación educativo-cultural, también es necesaria porque la nueva educación del siglo XXI, exige al profesor partir del conocimiento de la realidad socio-educativa y socio-cultural, basada en documentos nacionales como el CEPLAN, Proyecto Educativo Nacional 2021, y las Normas y Directivas de la Acreditación y Certificación Profesional de la Calidad Educativa ${ }^{28}$.

Respecto a los porcentajes alcanzados en las competencias específicas de los docentes, nos satisface porque la enseñanza de la nueva sociedad, se orienta a formar recursos humanos altamente calificados y ciudadanos responsables, capaces de atender a las necesidades de la vida humana ${ }^{29}$; y ello, sólo se puede lograr con dichas competencias específicas, apoyadas con las otras competencias genéricas y metódicas, respectivamente.

\section{CONCLUSIONES}

Del análisis realizado durante el desarrollo de esta investigación extraemos las siguientes conclusiones:

Se aprecia que en estudiantes sanmarquinos el 73\% (30) aplica el motivo cognitivometodológico; en egresados del mismo San Marcos el 80\% (93) aplica este mismo motivo cognitivo-metodológico en la elaboración del proyecto de tesis.

27 Ver la obra, La lógica de la investigación científica, de Karl Popper, pág 259.

28 Ver la obra, Modelo educativo San Marcos, pág. 18

29 Ver Misiones y funciones de la educación superior. Art.

$1^{\circ}$. Misión de educar, formar y realizar investigaciones

(UNESCO 1998). Ver Modelo educativo San Marcos, pág.24
Se observa que en estudiantes villarrealinos el $83 \%$ (72) aplica el motivo cognitivometodológico; en egresados del mismo Villarreal, el 85\% (125) aplican dicho motivo cognitivo-metodológico en la elaboración del proyecto de tesis.

Entretanto vemos que en estudiantes sanmarquinos el 63\% (26), aplica el motivo o factor motivacional-actitudinal; en egresados del mismo San Marcos el 68\% (79) aplica este mismo factor.

Del mismo modo se aprecia que en estudiantes villarrealinos el 79\% (69) aplica el motivo o factor motivacional-actitudinal; en egresados de esta institución aplica el 75\% (110) aplica dicho motivo.

Igualmente, observamos que los profesores sanmarquinos el $78 \%$ (32) aplican las competencias específicas, mientras que el $84 \%$ (73) de los profesores villarrealinos también utilizan estas mismas competencias específicas con las que propician la aplicación del modelo cuantitativo en la elaboración del proyecto de tesis.

\section{FUENTES DE INFORMACIÓN}

\section{Fuentes bibliográficas}

Aranzamendi, L. (2015). Investigación Jurídica. Segunda edición. Lima, Perú: Editorial Jurídica Grijley.

Aranzamendi, L. (2008). Epistemología y la Investigación Cualitativa y Cuantitativa en el Derecho. Primera Edición. Lima, Perú: Editorial ADRUS.

Barrantes Revoredo, Emilio (1950). Introducción a la Pedagogía. Primera Edición. Lima, Perú: Editorial Escuela Nueva S.A.

Fix Zamudio, Héctor (1995). Metodología, docencia e investigación jurídicas. Cuarta Edición. México, México: Editorial Porrúa, S.A.

Hernández Sampieri, Roberto y otros (2014). Metodología de la Investigación. México D.F., México: 600 pp.

Flores Barboza, J.C. (2013). Construyendo la tesis universitaria. Guía didáctica. Primera edición; Lima, Perú. Editorial San Marcos. 
Farro Custodio, Francisco (1999). Terminología básica de la investigación científica. Primera Edición. Lima, Perú: UDEGRAF S.A.

Fix Zamudio, Héctor. (1995). Metodología, docencia e investigación jurídicas. Cuarta Edición. México, México: Editorial Porrúa, S.A

Moragues Ribas de Pina, Mariano (1996). Hacia la escuela posible: sistematización del proyecto educativo del colegio La Casa de Cartón. Primera Edición. Lima-Perú: Tarea.

Noblecilla Morán, Ricardo (2006). Educación superior universitaria por competencias. Primera edición. Lima, Perú: INADEP.

OFICINA CENTRAL DE CALIDAD ACADÉMICA Y ACREDITACIÓN (OCCAA). Indicadores de Gestión para la UNMSM; 2005.

Popper Karl. 1962. La lógica de la investigación cientifica. Editorial TECNOS S.A. MadridEspaña, 451 pp.

Salazar Bondy, Augusto. 1965. En torno a la educación. Primera Edición. Editorial Universidad Nacional Mayor de San Marcos. Lima-Perú, 235 pp.

Salazar, M. 2006. Más allá de la excelencia y de la calidad. Tercera edición, México. Trillas. 292 páginas.

Sierra Bravo, R. 1984. Ciencias Sociales Epistemología, Lógica y Metodología. Teoría y ejercicios. Primera edición; Madrid, España; Paraninfo; 308 páginas.

Solís Espinoza, Alejandro (2008). Metodología de la investigación jurídico social, s/e; primera edición. Lima; 275 pp.

Tafur Portilla, R. 1995. La tesis universitaria. La tesis doctoral. La tesis de maestría. El informe. La monografía. Primera edición; Lima, Perú; Editorial Mantaro; 429 páginas.

Tamayo y Tamayo, Mario. 1984. Diccionario de la investigación científica. Editorial Limusa; primera edición. México; 242 Págs.

Unidad de Post Grado de la Facultad de Derecho. 2008. Monografias. Maestría y Doctorado. Primera edición; Jesús María, Perú; (spi); 279 páginas
UNIVERSIDAD NACIONAL MAYOR DE SAN MARCOS. Escuela de Post Grado. Maestrías y Doctorado. Prospecto 1999. 2000. Centro de Producción Editorial de la Universidad Nacional Mayor de San Marcos.

Valderrama Mendoza, Santiago. (2014). Pasos para elaborar proyectos de investigación científica. Segunda reimpresión. Lima, Perú: Editorial San Marcos.

VICERRECTORADO ACADÉMICO DE LA UNMSM. 2014. Guía Metódica de Diseño Curricular para Carreras de la UNMSM. Talleres Gráficos del Centro de Producción e Imprenta de la UNMSM., 63 pp,

VICERRECTORADO ACADÉMICO DE LA UNMSM. 2015. Jornadas Curriculares Universitarias 2013-2013. Talleres Gráficos del Centro de Producción e Imprenta de la UNMSM., 209 pp,

VICERRECTORADO ACADÉMICO DE LA UNMSM. 2015. Modelo de Responsabilidad Social de San Marcos. Talleres Gráficos del Centro de Producción e Imprenta de la UNMSM., 47 pp,

VICERRECTORADO ACADÉMICO DE LA UNMSM. 2015. Didáctica, Tutoría e Investigación Formativa. Talleres Gráficos del Centro de Producción e Imprenta de la UNMSM., 59 pp,

VICERRECTORADO ACADÉMICO DE LA UNMSM. 2015. Modelo Educativo San Marcos. Talleres Gráficos del Centro de Producción e Imprenta de la UNMSM., 115 pp,

VICERRECTORADO ACADÉMICO DE LA UNMSM. 2015. Tercer Encuentro de Docentes Sanmarquinos. Talleres Gráficos del Centro de Producción e Imprenta de la UNMSM., 35 pp,

Warren Howard C. Diccionario de Psicología (Compilador). (1996). Vigésima Segunda reimpresión. México, México: Fondo de cultura Económica.

Witker V. Jorge. (1987). Metodología de la Enseñanza del Derecho. Bogotá. Colombia: Editorial Temis

Witker V. Jorge. (1986). Cómo elaborar una tesis en Derecho. Pautas Metodológicas y Técnicas para el estudiante o investigador del 
Derecho. primera edición. Madrid, España: Editorial CIVITAS S.A.

\section{Fuente electrónicas}

Boletín $\mathrm{N}^{\circ} 45$. Editado por el Consejo Superior de Investigaciones de la UNMSM (marzomayo 2002).

La Producción Científica en San Marcos (1995-2001). Editado por el Consejo Superior de Investigaciones de Investigaciones y el Fondo Editorial de la UNMSM (2002).

Revista de Investigación. Editado por Unidad de Investigación de la Facultad de Derecho y Ciencia Política. UNMSM. Año $5-\mathrm{N}^{\circ} 7$ (noviembre 2003).

Unidad de Postgrado. UNMSM. Reglamento de Estudios y Evaluación de las Maestrías. Resolución Rectoral $\mathrm{N}^{\circ} 2200$-R-94, y restituida su vigencia por la Resolución Rectoral N 6921-R-01.

Fernández Muñoz, Ricardo (2003). Competencias profesionales del docente en la sociedad del siglo XXI. Recuperado del sitio de internet: ResearchGate https:// www.researchgate.net/profile/Ricardo Fernandez Munoz/publication/39153159 Competencias_profesionales_del docente_en_la_sociedad_del_siglo_XXI/

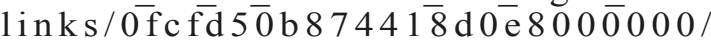
Competencias-profesionales-deldocente-en-la-sociedad-del-siglo-XXI. pdf?origin=publication_detail

\section{BIBLIOGRAFÍA COMPLEMENTARIA}

Angulo Argomedo, Jorge M. (1962). Enseñanza y Aprendizaje del Derecho. Trujillo, Perú: Faculta de Derecho de la Universidad Nacional de Trujillo.

Arista M. Gildomero L. (1980). Metodología de la investigación. Primera Edición. Lima, Perú: Editorial Albatros.

Cabanellas, G. (1989). Diccionario Enciclopédico de Derecho Usual. 21 ava edición. Buenos Aires, Argentina; Editorial Heliasta.

Carruitero Lecca, F. (2014). Introducción a la Metodología de la Investigación Jurídica. Primera edición. Lima, Perú: Ediciones Jurídicas San Bernardo.
Córdova Baldeón, I. (2008). Estadística Aplicada a la Investigación. Primera edición. Lima, Perú. Editorial San Marcos.

Cornejo, M. A. (2001). Lideres del Tercer Milenio la Revolución de los Valores. Primera edición. Naucalpan, México: Editorial Grad.

Farro Custodio, Francisco. (1999). Terminología básica de la Investigación científica. Primera Edición. Lima, Perú: UDEGRAF S.A.

Flores Barboza, J.C. (2013). Construyendo la Tesis Universitaria. Guía Didáctica. Primera edición. Lima, Perú: Editorial San Marcos.

Macchi, Luis. (1948). Diccionario de la Lengua Latina. Buenos Aires. Argentina: Sociedad Editora Internacional.

Masías Zavaleta, Demetrio. (2014). El Proceso de investigación científica. Primera Edición. Cusco, Perú: Impresiones Qosqo

Muñoz Bazo, c. (1998). Cómo elaborar y Asesorar una Investigación de Tesis. Primera edición; Naucalpán, México: Prentice Hall Hispanoamericana.

Ontoria Peña, Antonio y otro. (2005). Potenciar la capacidad de aprender a aprender. Primera Edición. Lima, Perú: Editorial Orbis Ventures SAC..

Pásara, L. (2004). La enseñanza del Derecho en el Perú: su impacto sobre la administración de Justicia. Primera edición; Lima, Perú: Ministerio de Justicia.

Ramos Núñez, Carlos (2000). Cómo hacer una tesis de Derecho y no envejecer en el intento. Primera edición. Lima: Gaceta Jurídica.

Ramos Suyo, J.A (2004). Elabore su Tesis en Derecho. Pre y PostGrado. Primera edición. Jesús María, Perú; Editorial San Marcos.

Ramos Núñez, C. (2005). Cómo hacer una Tesis de Derecho y no envejecer en el intento. Y cómo sustentar expedientes. Tercera edición. Miraflores, Perú: Gaceta Jurídica.

Quiroz Salazar, WILLIAM. (1998). La Investigación Jurídica. primera edición, Lima, Perú: IMSERGRAF EIRL. 
Robles, G. (1982). Epistemología y Derecho. Primera edición; Madrid, España: Editorial Pirámide.

Romero Quispe Jacobo (2011). Pasos de la investigación científica. Primera Edición. Lima, Perú: Imprenta Editorial Corporación Gráfica Aliaga S.A.C.
Rubio Correa, Marcial (2001). Ideas sobre qué es aprender (y enseñar) derecho en un pregrado. Lima, Perú: Fondo Editorial de la Pontificia Universidad Católica del Perú. 